\title{
Subclasses of Analytic Functions Defined by Generalized Hypergeometric Functions
}

\author{
Badr S. Alkahtani, ${ }^{1}$ Saima Mustafa, ${ }^{2}$ and Teodor Bulboacă ${ }^{3}$ \\ ${ }^{1}$ Mathematics Department, College of Science, King Saud University, P.O. Box 1142, Riyadh 11989, Saudi Arabia \\ ${ }^{2}$ Department of Mathematics, Pir Mehr Ali Shah Arid Agriculture University, Rawalpindi, Pakistan \\ ${ }^{3}$ Faculty of Mathematics and Computer Science, Babeş-Bolyai University, 400084 Cluj-Napoca, Romania
}

Correspondence should be addressed to Saima Mustafa; saimamustafa28@gmail.com

Received 2 September 2015; Revised 15 December 2015; Accepted 17 December 2015

Academic Editor: Alberto Fiorenza

Copyright (C) 2016 Badr S. Alkahtani et al. This is an open access article distributed under the Creative Commons Attribution License, which permits unrestricted use, distribution, and reproduction in any medium, provided the original work is properly cited.

We introduce a new subclass of analytic functions in the unit disk $\mathrm{U}$, defined by using the generalized hypergeometric functions, which extends some previous well-known classes defined by different authors. Inclusion results, radius problems, and some connections with the Bernardi-Libera-Livingston integral operator are discussed.

\section{Introduction}

Let $\mathscr{A}$ denote the class of functions $f$ of the form

$$
f(z)=z+\sum_{k=1}^{\infty} a_{k+1} z^{k+1}, \quad z \in \mathrm{U}
$$

which are analytic in the unit disk $\mathrm{U}$.

The convolution (or the Hadamard product) of two functions $f$ and $g$, where $f$ is given by (1) and $g(z)=z+$ $\sum_{k=1}^{\infty} b_{k+1} z^{k+1}, z \in \mathrm{U}$, is defined as

$$
(f * g)(z)=z+\sum_{k=1}^{\infty} a_{k+1} b_{k+1} z^{k+1}, \quad z \in \mathrm{U}
$$

For the complex parameters $\alpha_{i}$ with $\beta_{j} \in \mathbb{C} \backslash \mathbb{Z}_{0}^{-}$, where $\mathbb{Z}_{0}^{-}=\{0,-1,-2, \ldots\}, i \in\{1,2, \ldots, q\}$, and $j \in$ $\{1,2, \ldots, s\}$, we define the generalized hypergeometric function ${ }_{q} F_{s}\left(\alpha_{1}, \ldots, \alpha_{q} ; \beta_{1}, \ldots, \beta_{s} ; z\right)$ as follows (see $\left.[1,2]\right)$ :

$$
\begin{aligned}
& { }_{q} F_{s}\left(\alpha_{1}, \ldots, \alpha_{q} ; \beta_{1}, \ldots, \beta_{s} ; z\right)=\sum_{k=0}^{\infty} \frac{\left(\alpha_{1}\right)_{k} \cdots\left(\alpha_{q}\right)_{k}}{\left(\beta_{1}\right)_{k} \cdots\left(\beta_{s}\right)_{k}} \frac{z^{k}}{k !}, \\
& z \in \mathrm{U}, \quad\left(q \leq s+1 ; q, s \in \mathbb{N}_{0}=\mathbb{N} \cup\{0\} ; \mathbb{N}=\{1,2, \ldots\}\right),
\end{aligned}
$$

where $(\lambda)_{k}$ (or the shifted factorial) is defined by

$$
\begin{aligned}
& (\lambda)_{0}=1, \\
& (1)_{k}=k !, \quad k \in \mathbb{N}_{0}, \\
& (\lambda)_{k}=\lambda(\lambda+1) \cdots(\lambda+k-1), \quad k \in \mathbb{N} .
\end{aligned}
$$

Using the function $F\left(\alpha_{1}, \ldots, \alpha_{q} ; \beta_{1}, \ldots, \beta_{s} ; z\right)$ defined by

$$
\begin{aligned}
& F\left(\alpha_{1}, \ldots, \alpha_{q} ; \beta_{1}, \ldots, \beta_{s} ; z\right) \\
& \quad=z^{-m} \cdot{ }_{q} F_{s}\left(\alpha_{1}, \ldots, \alpha_{q} ; \beta_{1}, \ldots, \beta_{s} ; z\right),
\end{aligned}
$$

Liu and Srivastava [3] introduced and studied the properties of the linear operator $H\left(\alpha_{1}, \ldots, \alpha_{q} ; \beta_{1}, \ldots, \beta_{s}\right)$, defined by the Hadamard (or convolution) product

$$
\begin{aligned}
& H\left(\alpha_{1}, \ldots, \alpha_{q} ; \beta_{1}, \ldots, \beta_{s}\right) f(z) \\
& \quad=F\left(\alpha_{1}, \ldots, \alpha_{q} ; \beta_{1}, \ldots, \beta_{s} ; z\right) * f(z),
\end{aligned}
$$

where the function $f$ is analytic and $m$-valent in the punctured unit disk $\dot{U}=\mathrm{U} \backslash\{0\}$ and has the form $f(z)=z^{-m}+\sum_{k=1}^{\infty} a_{k} z^{k-m}$. Note that linear operator $H\left(\alpha_{1}, \ldots, \alpha_{q} ; \beta_{1}, \ldots, \beta_{s}\right)$ was motivated essentially by the work of Dziok and Srivastava [4]. 
Corresponding to the function $\mathscr{F}\left(\alpha_{1}, \ldots, \alpha_{q} ; \beta_{1}, \ldots, \beta_{s}\right.$; $z$ ) defined by

$$
\begin{aligned}
\mathscr{F} & \left(\alpha_{1}, \ldots, \alpha_{q} ; \beta_{1}, \ldots, \beta_{s} ; z\right) \\
& =z \cdot{ }_{q} F_{s}\left(\alpha_{1}, \ldots, \alpha_{q} ; \beta_{1}, \ldots, \beta_{s} ; z\right),
\end{aligned}
$$

we introduce a function $\mathscr{F}_{p}\left(\alpha_{1}, \ldots, \alpha_{q} ; \beta_{1}, \ldots, \beta_{s} ; z\right)$ given by

$$
\begin{aligned}
& \mathscr{F}\left(\alpha_{1}, \ldots, \alpha_{q} ; \beta_{1}, \ldots, \beta_{s} ; z\right) \\
& \quad * \mathscr{F}_{p}\left(\alpha_{1}, \ldots, \alpha_{q} ; \beta_{1}, \ldots, \beta_{s} ; z\right)=\frac{z}{(1-z)^{p}},
\end{aligned}
$$

where $p>0$.

Analogous to $H\left(\alpha_{1}, \ldots, \alpha_{q} ; \beta_{1}, \ldots, \beta_{s}\right)$ defined by (6), we now define the linear operator $H_{p}\left(\alpha_{1}, \ldots, \alpha_{q} ; \beta_{1}, \ldots, \beta_{s}\right)$ on $\mathscr{A}$ as follows:

$$
\begin{aligned}
& H_{p}\left(\alpha_{1}, \ldots, \alpha_{q} ; \beta_{1}, \ldots, \beta_{s}\right) f(z) \\
& \quad=\mathscr{F}_{p}\left(\alpha_{1}, \ldots, \alpha_{q} ; \beta_{1}, \ldots, \beta_{s} ; z\right) * f(z), \\
& \left(\alpha_{j}, \beta_{j} \in \mathbb{C} \backslash \mathbb{Z}_{0}^{-} ; i \in\{1,2, \ldots, q\} ; j \in\{1,2, \ldots, s\} ; p>0\right) .
\end{aligned}
$$

For convenience, we write

$$
H_{p, q, s}\left(\alpha_{1}\right):=H_{p}\left(\alpha_{1}, \ldots, \alpha_{q} ; \beta_{1}, \ldots, \beta_{s}\right) .
$$

Remark 1. The linear operator $H_{p}\left(\alpha_{1}, \ldots, \alpha_{q} ; \beta_{1}, \ldots, \beta_{s}\right)$ includes various other linear operators which were considered in some earlier works:

(i) In particular, for $p=1, q=2$, and $s=1$, we obtain the linear operator $H_{1}\left(\alpha_{1}, \alpha_{2} ; \beta_{1}\right)$ which was defined by Hohlov [5].

(ii) Moreover, putting $p=1, q=2, s=1$, and $\alpha_{2}=$ 1 , we obtain the well-known Carlson-Shaffer operator $L\left(\alpha_{1}, \beta_{1}\right)=H_{1}\left(\alpha, 1 ; \beta_{1}\right)($ see $[6,7])$.

From definitions (8) and (9), using notation (10), it is easy to prove the differentiation formula

$$
\begin{aligned}
z\left(H_{p, q, s}(\lambda) f(z)\right)^{\prime}= & p H_{p+1, q, s}(\lambda) f(z) \\
& -(p-1) H_{p, q, s}(\lambda) f(z) .
\end{aligned}
$$

We note that the operator $H_{p, q, s}(\lambda)$ is closely related to the Choi-Saigo [8] operator for analytic functions, which includes the integral studied by Liu [9] and K. I. Noor and M. A. Noor [10].

Let $\mathscr{P}_{k}(\rho)$ be the class of functions $h$, analytic in the unit disk $\mathrm{U}$, satisfying the condition $h(0)=1$ and

$$
\int_{0}^{2 \pi}\left|\frac{\operatorname{Re} h(z)-\rho}{1-\rho}\right| d \theta \leq k \pi,
$$

where $z=r e^{i \theta}, k \geq 2$, and $0 \leq \rho<1$. This class was introduced in [11], and as a special case we note that the class $\mathscr{P}_{k}(0)$ was defined by Pinchuk in [12]. Moreover, $\mathscr{P}(\rho)$ := $\mathscr{P}_{2}(\rho)$ is the class of analytic functions with the real part greater than $\rho$.
Remark 2. (i) Like in $[13,14]$, it can easily be seen that the function $h$, analytic in $\mathrm{U}$, with $h(0)=1$, belongs to $\mathscr{P}_{k}(\rho)$ if and only if there exists the functions $h_{1}, h_{2} \in \mathscr{P}(\rho)$ such that

$$
h(z)=\left(\frac{k}{4}+\frac{1}{2}\right) h_{1}(z)-\left(\frac{k}{4}-\frac{1}{2}\right) h_{2}(z), \quad z \in \mathrm{U} .
$$

(ii) It is known from [15] that the class $\mathscr{P}_{k}(\rho)$ is a convex set.

We will assume throughout our discussions, unless otherwise stated, that $\alpha_{i}, \beta_{j} \in \mathbb{C} \backslash \mathbb{Z}_{0}^{-}, i \in\{1,2, \ldots, q\}, j \in$ $\{1,2, \ldots, s\}$, with $q \leq s+1, p>0$, and all the powers represent the principal branches; that is, $\log 1=0$.

Using the linear operator $H_{p, q, s}(\lambda)$, we will define the following classes of analytic functions.

Definition 3. Let $0 \leq \rho<1, c\rangle 0$, and let $b$ be a complex number such that Re $b \geq 0$. A function $f \in \mathscr{A}$ is said to be in the class $H_{p, q, s}^{b}(\lambda, \rho, c, \delta, k)$ if and only if

$$
\begin{aligned}
& (1-b)\left[\frac{H_{p, q, s}(\lambda) f(z)}{H_{p, q, s}(\lambda) g(z)}\right]^{c} \\
& +b \frac{H_{p+1, q, s}(\lambda) f(z)}{H_{p+1, q, s}(\lambda) g(z)}\left[\frac{H_{p, q, s}(\lambda) f(z)}{H_{p, q, s}(\lambda) g(z)}\right]^{c-1} \\
& \in \mathscr{P}_{k}(\rho),
\end{aligned}
$$

where $k \geq 2$ and $g \in \mathscr{A}$ satisfies the condition

$$
\beta(z)=\frac{H_{p+1, q, s}(\lambda) g(z)}{H_{p, q, s}(\lambda) g(z)} \in \mathscr{P}(\delta), \quad 0 \leq \delta<1 .
$$

Remark 4. From the above definition, the following subclasses of $\mathscr{A}$ emerge as special cases:

(i) For $p=q=s=1, k=2, b=0, c=1, g(z)=z$, and $\lambda=1$, we have

$$
\begin{aligned}
& H_{1,1,1}^{0}(1, \rho, 1,0,2) \\
& \quad=\left\{f \in \mathscr{A}: \frac{f(z)}{z} \in \mathscr{P}(\rho), 0 \leq \rho<1\right\},
\end{aligned}
$$

and this class was studied by Chen [16].

(ii) When $p=q=s=1, g(z)=z$, and $\lambda=1$, then $H_{1,1,1}^{b}(1, \rho, c, \delta, k)$ reduces to the class studied by Noor [13].

(iii) For $p=q=s=1, b=1, k=2, g(z)=z$, and $\lambda=1$, we obtain the class

$$
\begin{aligned}
& H_{1,1,1}^{1}(1, \rho, c, 0,2)=\left\{f \in \mathscr{A}: f^{\prime}(z)\left(\frac{f(z)}{z}\right)^{c-1}\right. \\
& \quad \in \mathscr{P}(\rho), 0 \leq \rho<1\},
\end{aligned}
$$

which was studied by Ponnusamy and Karunakaran [17]. 
We will use the following lemmas to prove our main results.

Lemma 5 (see [18]). If $h$ is an analytic function in $\mathrm{U}$, with $h(0)=1$, and if $\lambda_{1}$ is a complex number satisfying $\operatorname{Re} \lambda_{1} \geq 0$, $\lambda_{1} \neq 0$, then

$$
\operatorname{Re}\left[h(z)+\lambda_{1} z h^{\prime}(z)\right]>\rho, \quad z \in \mathrm{U}, \quad(0 \leq \rho<1)
$$

implies

$$
\operatorname{Re} h(z)>\gamma, \quad z \in \mathrm{U},
$$

where $\gamma$ is given by

$$
\begin{aligned}
\gamma & =\rho+(1-\rho)\left(2 \gamma_{1}-1\right), \\
\gamma_{1} & =\int_{0}^{1}\left(1+t^{\operatorname{Re} \lambda_{1}}\right)^{-1} d t,
\end{aligned}
$$

and $\gamma_{1}$ is an increasing function of $\operatorname{Re} \lambda_{1}$, and $1 / 2 \leq \gamma_{1}<1$. The estimate is sharp in the sense that the bound cannot be improved.

Lemma 6 (see [19]). Let $u=u_{1}+i u_{2}, v=v_{1}+i v_{2}$, and let $\psi(u, v)$ be a complex-valued function satisfying the following conditions:

(i) $\psi(u, v)$ is continuous in a domain $D \subset \mathbb{C}^{2}$.

(ii) $(1,0) \in D$ and $\operatorname{Re} \psi(1,0)>0$.

(iii) $\operatorname{Re} \psi\left(i u_{2}, v_{1}\right) \leq 0$, whenever $\left(i u_{2}, v_{1}\right) \in D$ and $v_{1} \leq$ $-(1 / 2)\left(1+u_{2}^{2}\right)$.

If $p(z)=1+\sum_{m=1}^{\infty} c_{m} z^{m}$ is an analytic function in $\mathrm{U}$, such that $\left(p(z), z p^{\prime}(z)\right) \in D$ for all $z \in \mathrm{U}$, and $\operatorname{Re} \psi\left(p(z), z p^{\prime}(z)\right)>$ 0 for all $z \in \mathrm{U}$, then $\operatorname{Re} p(z)>0$ for all $z \in \mathrm{U}$.

In this paper, we investigate several properties of the class $H_{p, q, s}^{b}(\lambda, \rho, c, \delta, k)$, like inclusion results and radius problems; moreover, a connection with the Bernardi-Libera-Livingston integral operator is also discussed.

\section{Main Results}

Theorem 7. If $b \geq 0$, then $H_{p, q, s}^{b}(\lambda, \rho, c, \delta, k) \subset H_{p, q, s}^{0}(\lambda$, $\rho, c, \delta, k)$.

Proof. Let an arbitrary function $f \in H_{p, q, s}^{b}(\lambda, \rho, c, \delta, k)$, and denote

$$
h(z):=\left(\frac{H_{p, q, s}(\lambda) f(z)}{H_{p, q, s}(\lambda) g(z)}\right)^{c}
$$

where $h$ is analytic in $\mathrm{U}$, with $h(0)=1$, and $g$ satisfies condition (15). From part (i) of Remark 2, we have that $f \in$ $H_{p, q, s}^{0}(\lambda, \rho, c, \delta, k)$, if and only if

$$
h(z)=\left(\frac{k}{4}+\frac{1}{2}\right) h_{1}(z)-\left(\frac{k}{4}-\frac{1}{2}\right) h_{2}(z),
$$

where $h_{1}, h_{2} \in \mathscr{P}(\rho)$.
Using the differentiation formula (11) together with (15), after an elementary computation, we obtain

$$
\begin{aligned}
& (1-b)\left[\frac{H_{p, q, s}(\lambda) f(z)}{H_{p, q, s}(\lambda) g(z)}\right]^{c} \\
& +b \frac{H_{p+1, q, s}(\lambda) f(z)}{H_{p+1, q, s}(\lambda) g(z)}\left[\frac{H_{p, q, s}(\lambda) f(z)}{H_{p, q, s}(\lambda) g(z)}\right]^{c-1} \\
& =h(z)+\frac{b z h^{\prime}(z)}{p c \beta(z)},
\end{aligned}
$$

where $\beta$ is given by (15).

Now, using the representation formula (22), we have

$$
\begin{aligned}
h(z)+\frac{b z h^{\prime}(z)}{p c \beta(z)}= & \left(\frac{k}{4}+\frac{1}{2}\right)\left[h_{1}(z)+\frac{b z h_{1}^{\prime}(z)}{p c \beta(z)}\right] \\
& -\left(\frac{k}{4}-\frac{1}{2}\right)\left[h_{2}(z)+\frac{b z h_{2}^{\prime}(z)}{p c \beta(z)}\right] .
\end{aligned}
$$

Since $f \in H_{p, q, s}^{b}(\lambda, \rho, c, \delta, k)$, from relations (23) and (24), it follows that

$$
h_{i}(z)+\frac{b z h_{i}^{\prime}(z)}{p c \beta(z)} \in \mathscr{P}(\rho), \quad i=1,2,
$$

and using the substitution $H_{i}(z):=h_{i}(z)-\rho, i=1,2$, the above relation becomes

$$
H_{i}(z)+\frac{b z H_{i}^{\prime}(z)}{p c \beta(z)} \in \mathscr{P}(0), \quad i=1,2
$$

To prove our result, we need to show that (26) implies $H_{i} \in \mathscr{P}(0), i=1,2$. We will define the functional $\psi(u, v)$ by taking $u=H_{i}(z)$, and $v=z H_{i}^{\prime}(z)$, and thus we have

$$
\psi(u, v)=u+\frac{b v}{p c \beta(z)}
$$

It is easy to see that the first two conditions of Lemma 6 are satisfied; hence, we proceed to verify condition (iii). Since $\beta \in \mathscr{P}(\delta)$, that is, $\operatorname{Re} \beta(z)>\delta$ for all $z \in \mathrm{U}, 0 \leq \delta<1$, it follows that

$$
\operatorname{Re} \psi\left(i u_{2}, v_{1}\right)=\operatorname{Re} \frac{b v_{1}}{p c \beta(z)} \leq-\frac{b\left(1+u_{2}^{2}\right) \delta}{2 p c|\beta(z)|^{2}} \leq 0
$$

whenever $v_{1} \leq-(1 / 2)\left(1+u_{2}^{2}\right)$. Using Lemma 6 , we conclude that $H_{i} \in \mathscr{P}(0)$, for $i=1,2$, which completes our proof.

Theorem 8. If $0 \leq b_{1}<b_{2}$, then $H_{p, q, s}^{b_{2}}(\lambda, \rho, c, \delta, k) \subset H_{p, q, s}^{b_{1}}(\lambda$, $\rho, c, \delta, k)$. 
Proof. If we consider an arbitrary function $f \in H_{p, q, s}^{b_{2}}(\lambda, \rho$, $c, \delta, k)$, then $\varphi_{2} \in \mathscr{P}_{k}(\rho)$, where

$$
\begin{aligned}
& \varphi_{2}(z) \\
& \quad:=\left(1-b_{2}\right)\left[\frac{H_{p, q, s}(\lambda) f(z)}{H_{p, q, s}(\lambda) g(z)}\right]^{c} \\
& \quad+b_{2} \frac{H_{p+1, q, s}(\lambda) f(z)}{H_{p+1, q, s}(\lambda) g(z)}\left[\frac{H_{p, q, s}(\lambda) f(z)}{H_{p, q, s}(\lambda) g(z)}\right]^{c-1} .
\end{aligned}
$$

According to Theorem 7, we have

$$
\varphi_{1}(z):=\left(\frac{H_{p, q, s}(\lambda) f(z)}{H_{p, q, s}(\lambda) g(z)}\right)^{c} \in \mathscr{P}_{k}(\rho),
$$

and a simple computation shows that

$$
\begin{aligned}
& \left(1-b_{1}\right)\left[\frac{H_{p, q, s}(\lambda) f(z)}{H_{p, q, s}(\lambda) g(z)}\right]^{c} \\
& \quad+b_{1} \frac{H_{p+1, q, s}(\lambda) f(z)}{H_{p+1, q, s}(\lambda) g(z)}\left[\frac{H_{p, q, s}(\lambda) f(z)}{H_{p, q, s}(\lambda) g(z)}\right]^{c-1} \\
& =\left(1-\frac{b_{1}}{b_{2}}\right) \varphi_{1}(z)+\frac{b_{1}}{b_{2}} \varphi_{2}(z) .
\end{aligned}
$$

Since the class $\mathscr{P}_{k}(\rho)$ is a convex set (see (ii) from Remark 2), it follows that the right-hand side of (31) belongs to $\mathscr{P}_{k}(\rho)$ for $0 \leq b_{1}<b_{2}$, which implies that $f \in H_{p, q, s}^{b_{1}}(\lambda, \rho, c, \delta, k)$.

Now, let us define the operator $J_{c}: \mathscr{A} \rightarrow \mathscr{A}$ by

$$
J_{c} f(z)=\frac{c+1}{z^{c}} \int_{0}^{z} t^{c-1} f(t) d t \quad(c>-1) .
$$

For $c \in \mathbb{N}$, the operator $J_{c}$ was introduced by Bernardi [20], while the special case $J_{1}$ was previously studied by Libera [21] and Livingston [22].

Theorem 9. If $f \in \mathscr{A}, J_{c}(f)$ is given by (32), and $b \in \mathbb{C}$ with $\operatorname{Re} b>0$, then

$$
(1-b) \frac{H_{p, q, s}(\lambda) J_{c} f(z)}{z}+b \frac{H_{p, q, s}(\lambda) f(z)}{z} \in \mathscr{P}_{k}(\rho)
$$

implies that

$$
\frac{H_{p, q, s}(\lambda) J_{c} f(z)}{z} \in \mathscr{P}_{k}(\gamma),
$$

where $\gamma$ is given by (20), with $\lambda_{1}=b /(c+1)$.

Proof. Differentiating relation (32), we have

$$
z\left(J_{c} f(z)\right)^{\prime}=(c+1) f(z)-c J_{c} f(z),
$$

and using definition (9), this implies

$$
\begin{aligned}
z\left(H_{p, q, s}(\lambda) J_{c} f(z)\right)^{\prime}= & (c+1) H_{p, q, s}(\lambda) f(z) \\
& -c H_{p, q, s}(\lambda) J_{c} f(z) .
\end{aligned}
$$

If we let

$$
h(z):=\frac{H_{p, q, s}(\lambda) J_{c} f(z)}{z}
$$

according to part (i) of Remark 2, we need to prove that $h$ is of the form

$$
h(z)=\left(\frac{k}{4}+\frac{1}{2}\right) h_{1}(z)-\left(\frac{k}{4}-\frac{1}{2}\right) h_{2}(z),
$$

where $h_{1}, h_{2} \in \mathscr{P}(\gamma)$.

Using (36), from the above relation, we have

$$
\begin{aligned}
(1-b) & \frac{H_{p, q, s}(\lambda) J_{c} f(z)}{z}+b \frac{H_{p, q, s}(\lambda) f(z)}{z} \\
= & h(z)+\frac{b z h^{\prime}(z)}{c+1} \\
= & \left(\frac{k}{4}+\frac{1}{2}\right)\left[h_{1}(z)+\frac{b z h_{1}^{\prime}(z)}{c+1}\right] \\
& -\left(\frac{k}{4}-\frac{1}{2}\right)\left[h_{2}(z)+\frac{b z h_{2}^{\prime}(z)}{c+1}\right] \in \mathscr{P}_{k}(\rho) .
\end{aligned}
$$

Thus, from part (i) of Remark 2, it follows that

$$
h_{i}(z)+\frac{b z h_{i}^{\prime}(z)}{c+1} \in \mathscr{P}(\rho), \quad i=1,2,
$$

and from Lemma 5, we conclude that $h_{i} \in \mathscr{P}(\gamma), i=1,2$, with $\gamma$ given by $(20)$ and $\lambda_{1}=b /(c+1)$.

The next result deals with the converse of Theorem 7.

Theorem 10. Let $b>0$ and $0<\delta<1$. If $f \in$ $H_{p, q, s}^{0}(\lambda, \rho, c, \delta, k)$, then $f \in H_{p, q, s}^{b}(\lambda, \rho, c, \delta, k)$ for $|z|<R$, where

$$
\begin{aligned}
& R=\min \left\{\frac{-b+\sqrt{b^{2}+p^{2} c^{2} \delta^{2}}}{p c \delta} ;\right. \\
& \left.\frac{p c \rho \delta-b+\sqrt{(p c \rho \delta-b)^{2}+p^{2} c^{2} \delta^{2}\left(1-\rho^{2}\right)}}{p c \delta(1-\rho)}\right\} .
\end{aligned}
$$

Proof. For arbitrary $f \in H_{p, q, s}^{0}(\lambda, \rho, c, \delta, k)$, let us define the function $h$ as in (43). Thus, it follows that

$$
\left(\frac{H_{p, q, s}(\lambda) f(z)}{H_{p, q, s}(\lambda) g(z)}\right)^{c}=h(z) \in \mathscr{P}_{k}(\rho)
$$

and $g \in \mathscr{A}$ satisfies the condition

$$
\beta(z)=\frac{H_{p+1, q, s}(\lambda) g(z)}{H_{p, q, s}(\lambda) g(z)} \in \mathscr{P}(\delta)
$$


From part (i) of Remark 2, we have that (42) holds if and only if

$$
h(z)=\left(\frac{k}{4}+\frac{1}{2}\right) h_{1}(z)-\left(\frac{k}{4}-\frac{1}{2}\right) h_{2}(z),
$$

where $h_{1}, h_{2} \in \mathscr{P}(\rho)$.

Using the above representation formula, similar to the proof of Theorem 7, we deduce that

$$
\begin{aligned}
(1-b) & {\left[\frac{H_{p, q, s}(\lambda) f(z)}{H_{p, q, s}(\lambda) g(z)}\right]^{c} } \\
+ & b \frac{H_{p+1, q, s}(\lambda) f(z)}{H_{p+1, q, s}(\lambda) g(z)}\left[\frac{H_{p, q, s}(\lambda) f(z)}{H_{p, q, s}(\lambda) g(z)}\right]^{c-1} \\
= & \left(\frac{k}{4}+\frac{1}{2}\right)\left[h_{1}(z)+\frac{b z h_{1}^{\prime}(z)}{p c \beta(z)}\right] \\
& -\left(\frac{k}{4}-\frac{1}{2}\right)\left[h_{2}(z)+\frac{b z h_{2}^{\prime}(z)}{p c \beta(z)}\right],
\end{aligned}
$$

and substituting $H_{i}(z):=h_{i}(z)-\rho, i=1,2$, we finally obtain

$$
\begin{aligned}
(1-b) & {\left[\frac{H_{p, q, s}(\lambda) f(z)}{H_{p, q, s}(\lambda) g(z)}\right]^{c} } \\
+ & b \frac{H_{p+1, q, s}(\lambda) f(z)}{H_{p+1, q, s}(\lambda) g(z)}\left[\frac{H_{p, q, s}(\lambda) f(z)}{H_{p, q, s}(\lambda) g(z)}\right]^{c-1} \\
= & \left(\frac{k}{4}+\frac{1}{2}\right)\left[H_{1}(z)+\rho+\frac{b z H_{1}^{\prime}(z)}{p c \beta(z)}\right] \\
& -\left(\frac{k}{4}-\frac{1}{2}\right)\left[H_{2}(z)+\rho+\frac{b z H_{2}^{\prime}(z)}{p c \beta(z)}\right]
\end{aligned}
$$

where $H_{1}, H_{2} \in \mathscr{P}(0)$.

To prove our result, we need to determine the value of $R$, such that

$$
\begin{aligned}
\operatorname{Re}\left[H_{i}(z)+\rho+\frac{b z H_{i}^{\prime}(z)}{p c \beta(z)}\right]> & 0, \\
& \text { for }|z|<R, i=1,2,
\end{aligned}
$$

whenever $H_{1}, H_{2} \in \mathscr{P}(0)$.

Using the well-known estimates for the class $\mathscr{P}(0)$ [23], that is,

$$
\begin{gathered}
\left|H_{i}^{\prime}(z)\right| \leq \frac{2 \operatorname{Re} H_{i}(z)}{1-r^{2}}, \quad|z| \leq r<1, \quad i=1,2, \\
\operatorname{Re} H_{i}(z) \geq \frac{1-r}{1+r}, \quad|z| \leq r<1, \quad i=1,2,
\end{gathered}
$$

and according to (43), we obtain

$$
\begin{aligned}
& \operatorname{Re}\left[H_{i}(z)+\rho+\frac{b z H_{i}^{\prime}(z)}{p c \beta(z)}\right] \\
& \geq \rho+\operatorname{Re} H_{i}(z)-\frac{b}{p c} \frac{\left|z H_{i}^{\prime}(z)\right|}{|\beta(z)|} \\
& \geq \rho+\operatorname{Re} H_{i}(z)-\frac{b}{p c} \frac{\left|z H_{i}^{\prime}(z)\right|}{\delta} \\
& \geq \rho+\operatorname{Re} H_{i}(z)\left[1-\frac{2 b}{p c \delta} \frac{r}{1-r^{2}}\right],
\end{aligned}
$$

for all $|z| \leq r<1$ and $i=1,2$.

A simple computation shows that $1-(2 b / p c \delta)(r /(1-$ $\left.\left.r^{2}\right)\right) \geq 0(0 \leq r<1)$ if and only if

$$
r \in\left[0, \frac{-b+\sqrt{b^{2}+p^{2} c^{2} \delta^{2}}}{p c \delta}\right] .
$$

Assuming that (50) holds, from (49), we deduce that

$$
\begin{aligned}
& \operatorname{Re}\left[H_{i}(z)+\rho+\frac{b z H_{i}^{\prime}(z)}{p c \beta(z)}\right] \\
& \quad \geq \rho+\frac{1-r}{1+r}\left[1-\frac{2 b}{p c \delta} \frac{r}{1-r^{2}}\right], \quad|z| \leq r<1,
\end{aligned}
$$

for $i=1,2$. It is easy to see that the right-hand side of the above inequality is greater than or equal to zero if and only if

$$
\begin{aligned}
& r \in[0, \min \{1 ; \\
& \left.\left.\frac{p c \rho \delta-b+\sqrt{(p c \rho \delta-b)^{2}+p^{2} c^{2} \delta^{2}\left(1-\rho^{2}\right)}}{p c \delta(1-\rho)}\right\}\right],
\end{aligned}
$$

and combining this with (50), we obtain our result.

Remark 11. We note the following special case obtained from the above result: for $\rho=0$, formula (41) reduces to

$$
R=\frac{-b+\sqrt{b^{2}+p^{2} c^{2} \delta^{2}}}{p c \delta}
$$

\section{Conflict of Interests}

The authors declare that there is no conflict of interests regarding the publication of this paper.

\section{Acknowledgments}

The work of the third author was entirely supported by the grant given by Babeş-Bolyai University, dedicated for 
Supporting the Excellence Research 2015. The first author, Badr S. Alkahtani, is grateful to King Saud University, Deanship of Scientific Research, College of Science Research Center, for supporting this project.

\section{References}

[1] S. Owa and H. M. Srivastava, "Univalent and starlike generalized hypergeometric functions," Canadian Journal of Mathematics, vol. 39, no. 5, pp. 1057-1077, 1987.

[2] H. M. Srivastava and S. Owa, "Some characterization and distortion theorems involving fractional calculus, generalized hypergeometric functions, Hadamard products, linear operators, and certain subclasses of analytic functions," Nagoya Mathematical Journal, vol. 106, pp. 1-28, 1987.

[3] J.-L. Liu and H. M. Srivastava, "Classes of meromorphically multivalent functions associated with the generalized hypergeometric function," Mathematical and Computer Modelling, vol. 39, no. 1, pp. 21-34, 2004.

[4] J. Dziok and H. M. Srivastava, "Certain subclasses of analytic functions associated with the generalized hypergeometric function," Integral Transforms and Special Functions, vol. 14, no. 1, pp. 7-18, 2003.

[5] Yu. E. Hohlov, "Operators and operations in the class of univalent functions," Izvestiya Vysshikh Uchebnykh Zavedenii. Matematika, vol. 10, pp. 83-89, 1978.

[6] B. C. Carlson and D. B. Shaffer, "Starlike and prestarlike hypergeometric functions," SIAM Journal on Mathematical Analysis, vol. 15, no. 4, pp. 737-745, 1984.

[7] K. I. Noor, S. Mustafa, and B. Malik, "On some classes of $p$ valent functions involving Carlson-Shaffer operator," Applied Mathematics and Computation, vol. 214, no. 2, pp. 336-341, 2009.

[8] J. H. Choi, M. Saigo, and H. M. Srivastava, "Some inclusion properties of a certain family of integral operators," Journal of Mathematical Analysis and Applications, vol. 276, no. 1, pp. 432445, 2002.

[9] J.-L. Liu, "The Noor integral and strongly starlike functions," Journal of Mathematical Analysis and Applications, vol. 261, no. 2, pp. 441-447, 2001.

[10] K. I. Noor and M. A. Noor, “On integral operators," Journal of Mathematical Analysis and Applications, vol. 238, no. 2, pp. 341352, 1999.

[11] K. S. Padmanabhan and R. Parvatham, "Properties of a class of functions with bounded boundary rotation," Annales Polonici Mathematici, vol. 31, no. 3, pp. 311-323, 1975.

[12] B. Pinchuk, "Functions of bounded boundary rotation," Israel Journal of Mathematics, vol. 10, pp. 6-16, 1971.

[13] K. I. Noor, "On certain classes of analytic functions," Journal of Inequalities in Pure and Applied Mathematics, vol. 7, no. 2, article 49, 2006.

[14] K. I. Noor, "On analytic functions related to certain family of integral operators," Journal of Inequalities in Pure and Applied Mathematics, vol. 7, no. 2, article 69, 2006.

[15] K. I. Noor, "On subclasses of close-to-convex functions of higher order," International Journal of Mathematics and Mathematical Sciences, vol. 15, no. 2, pp. 279-289, 1992.

[16] M. P. Chen, "On the regular functions satisfying $\operatorname{Re}[f(z) / z]>$ $\alpha$, Bulletin of the Institute of Mathematics Academia Sinica New Series, vol. 3, pp. 65-70, 1975.
[17] S. Ponnusamy and V. Karunakaran, "Differential subordination and conformal mappings," Complex Variables, Theory and Application, vol. 11, no. 2, pp. 79-86, 1989.

[18] S. Ponnusamy, "Differential sobordination and Bazilevič functions," Proceedings of the Indian Academy of Sciences, vol. 105, no. 2, pp. 169-186, 1995.

[19] S. S. Miller, "Differential inequalities and Carathéodory functions," Bulletin of the American Mathematical Society, vol. 81, pp. 79-81, 1975.

[20] S. D. Bernardi, "Convex and starlike univalent functions," Transactions of the American Mathematical Society, vol. 135, pp. 429-446, 1969.

[21] R. J. Libera, "Some radius of convexity problems," Duke Mathematical Journal, vol. 31, pp. 143-158, 1964.

[22] A. E. Livingston, "On the radius of univalence of certain analytic functions," Proceedings of the American Mathematical Society, vol. 17, pp. 352-357, 1966.

[23] A. W. Goodman, Univalent Functions, Volumes I \& II, Polygonal Publishing House, Washington, NJ, USA, 1983. 


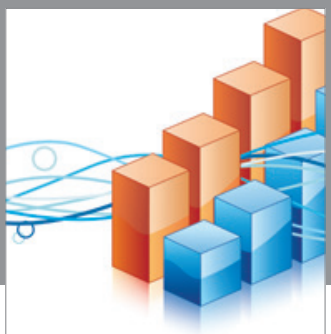

Advances in

Operations Research

vatem alat4

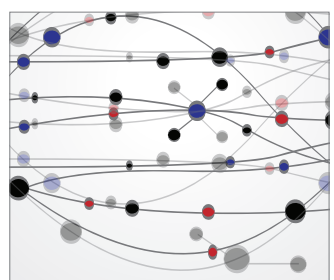

\section{The Scientific} World Journal
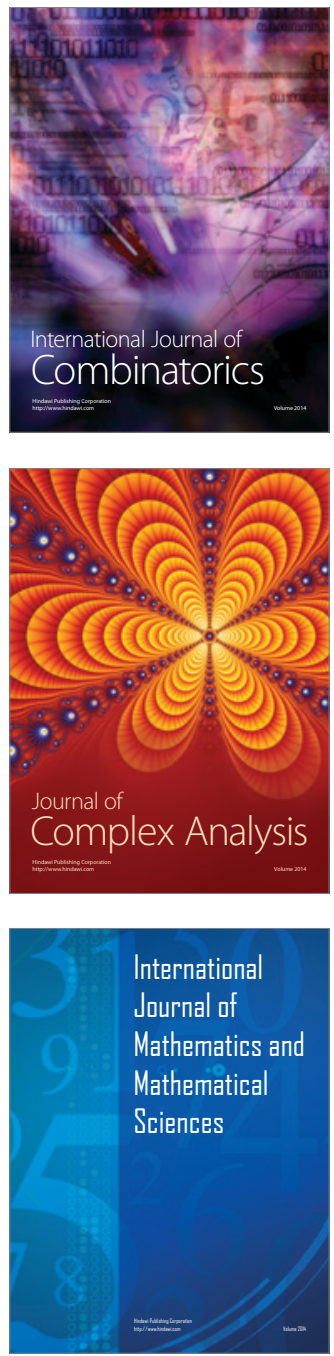
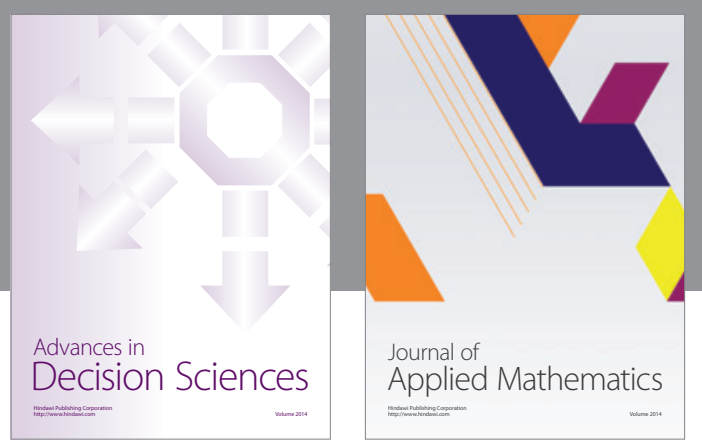

Algebra

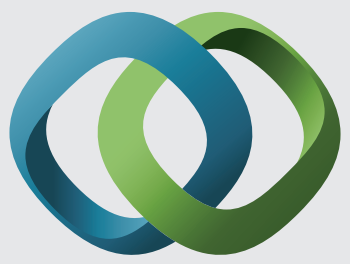

\section{Hindawi}

Submit your manuscripts at

http://www.hindawi.com
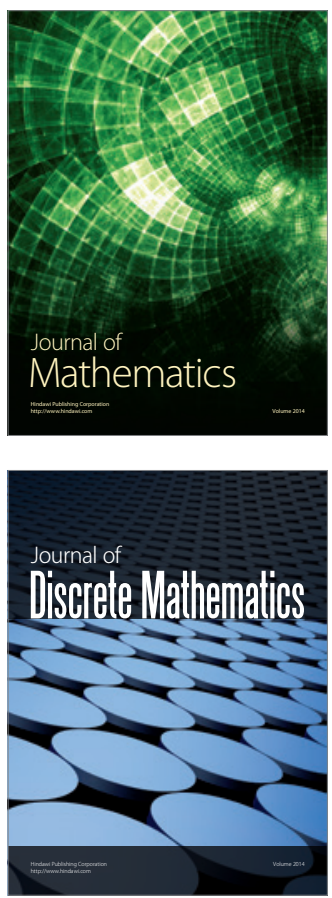

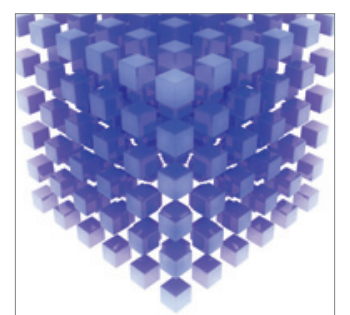

Mathematical Problems in Engineering
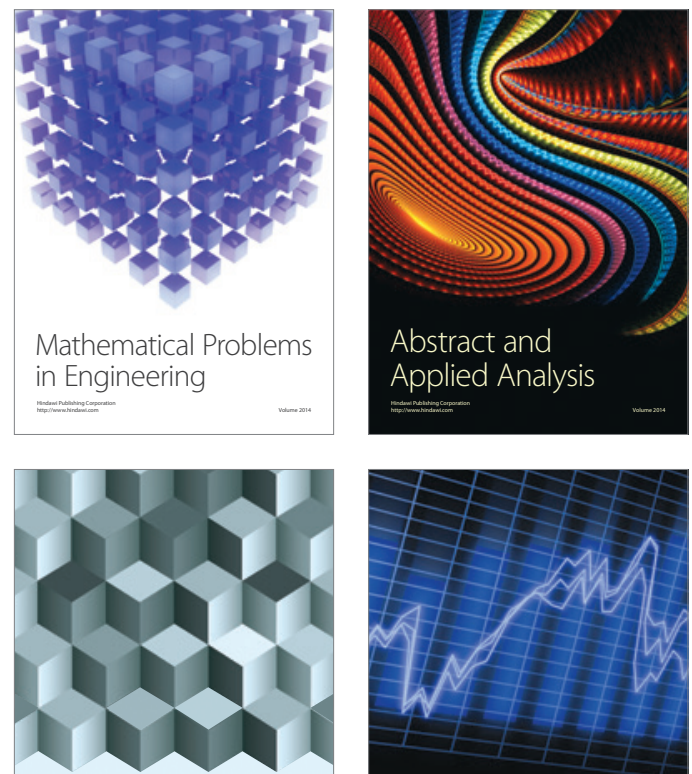

Journal of

Function Spaces

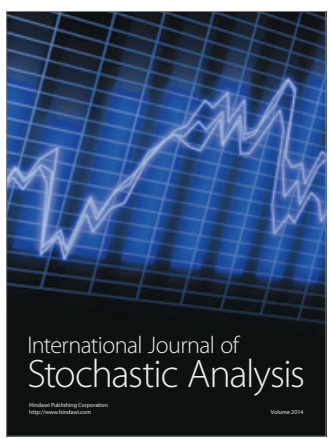

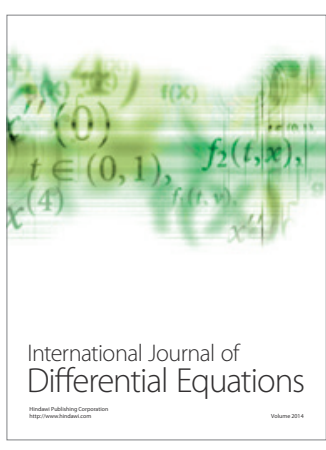
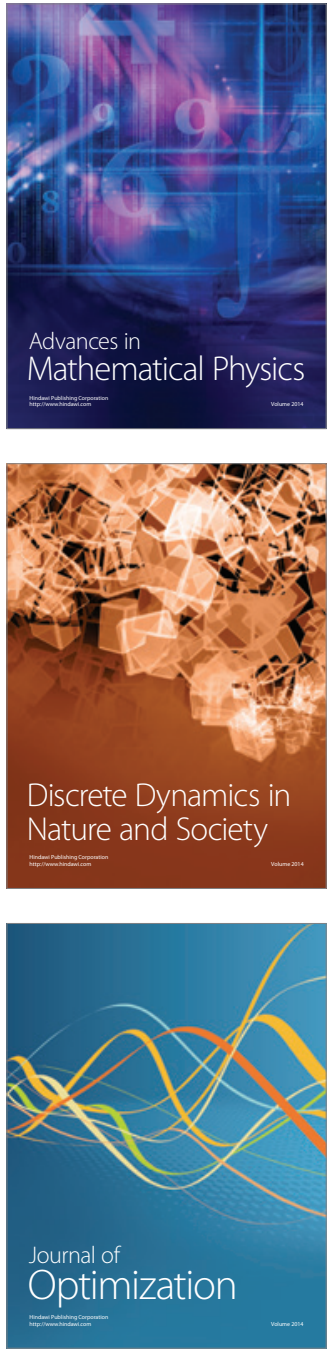